\title{
Ein (nicht ganz so) klarer Fall
}

\section{Eine junge Patientin mit THERAPIERESISTENTER ANÄMIE ohne bekannte Ursache gibt Rätsel auf: Welche Erkrankung ist noch im Gepäck? Claus Jahn}

Der Fall schien schon in dem Moment klar zu sein, als die junge Frau das Behandlungszimmer betrat. Ihr Erscheinungsbild war so typisch! Ihre Blässe ließ an die Redensart denken: „so weiß, wie eine Wand“. Ihre dunklen Augenringe, auffällig helle Konjunktiven und fast schon bläulich-livide verfärbte Lippen rundeten das Bild ab. Hinzu kamen noch ein müder, erschöpfter Gesichtsausdruck und ein entsprechend schleppendes Gangbild. Mich hätte es sehr gewundert, wenn die 18-Jährige nicht von den Beschwerden einer manifesten Anämie gezeichnet gewesen wäre. Kaum, dass sie sich gesetzt hatte, fielen mir auch noch die ebenfalls blutleeren Nägel mit ihrer bläulich-weißen Lunula auf.

\section{KURZ GEFASST}

1 Mit typischen Anämiezeichen stellt sich eine junge, sportliche Patientin vor, darunter Blässe, Erschöpfung, Atemnot, Herzklopfen sowie erniedrigte Eisen, $\mathrm{Hb}$ - und Hämatokritwerte.

2

Wie sich zeigt, ist exzessiver Sport ursächlich beteiligt, aber auch die bislang maskierte und unentdeckte Kupferspeicherkrankheit Morbus Wilson.

3

Mit einer Kombination aus alpha-Liponsäure, Zink, Eisen,

Vitamin $B_{12}$, Folsäure, Lebensstil- und Ernährungsumstellung bessert sich der Zustand innerhalb von acht Wochen erheblich.
Das gesamte Bild passte. Dazu kam, dass Sophie P. (Name von der Redaktion geändert) mit $158 \mathrm{~cm}$ und $46 \mathrm{~kg}$ recht klein und zierlich war. Ihr mit sehr kalten Fingern ausgeführter, trockener Händedruck bewies aber eine unvermutete Kraft.

\section{Verdacht auf Anämie durch alle Zeichen bestätigt}

Doch bevor ich mich zu voreilig auf ein Krankheitsbild festlege, steht immer die Anamnese an. Hierbei ist es ein Grundsatz für mich, möglichst unvoreingenommen zu sein und meine Spontan- 
diagnosen erst einmal nur im Hinterkopf zu behalten. Nichts ist schlimmer als eine vorgefertigte Meinung, für die man dann im weiteren Verlauf passende Symptome und Hinweise sucht. Nicht selten führt genau das zu unzureichenden oder sogar falschen Diagnosen.

Die Anamnese bestätigte meine Verdachtsdiagnose jedoch nochmals: Sophie P. klagte über ein deutliches Leistungsdefizit, zum Teil mit massiven Schwitzattacken, die für sie sehr untypisch seien. Auch leide sie unter Müdigkeit bis hin zu Erschöpfung, manchmal schon gleich nach dem Aufstehen am Morgen. Ein Mittagsschlaf helfe nicht. Wenn sie sich einmal leistungsfähig fühle, dann ermüde sie unglaublich schnell. Alle Symptome schienen in Wellen zu verlaufen, was sie nicht zuordnen konnte. Dazu zählte auch Atemnot (Dyspnoe) schon bei leichten Belastungen, obwohl sie sehr sportlich war. Oft schlage ihr das Herz rasend schnell in der Brust.

$\rightarrow$ Alle Symptome passen zur Diagnose Anämie, darunter Blässe, Leistungsdefizit, Atemnot, Schwindel und Kopfschmerzen. Auch die Tachykardie ist typisch: Hierbei versucht der Organismus, durch den erhöhten Herzschlag die fehlende Sauerstofftransportkapazität der Erythrozyten wettzumachen und die Versorgung aufrechtzuerhalten. Dass die Symptome erst seit einigen Monaten auftreten, fügt sich ebenfalls in das Bild ein.
Die Patientin berichtete auch über Schwindel, der vor allem bei Lagewechseln einsetze (orthostatischer Schwindel), zunehmend aber auch „einfach so“. Manchmal habe sie Angst, ohnmächtig zu werden. Kopfschmerzen hatte sie bis vor wenigen Monaten praktisch nie, jetzt aber fast täglich. Sie erwähnte auch Konzentrationsschwierigkeiten, die sie auf die Cephalgie zurückführe. Aber auch Vergesslichkeit und eine für sie untypische Teilnahmslosigkeit hätten sich zunehmend entwickelt.

Damit lieferte sie eine pschyrembeltaugliche Beschreibung der Anämie. $\mathrm{Zu}$ fehlen schienen nur noch die manchmal auftretende Schlaflosigkeit, Tinnitus, brüchige Nägel und splissige Haare. Sophie P. berichtete, dass sie bis vor neun oder zehn Monaten keine Probleme gehabt habe. Nun befand sie sich jedoch am vorläufigen Endpunkt eines für sie belastenden Praxismarathons.

\section{Laborwerte untermauern Diagnose, doch etwas stört}

Wie nach zahlreichen Praxisbesuchen nicht weiter verwunderlich, konnte Sophie P. eine Fülle von ärztlichen Befundungen vorweisen. Zunächst war wie üblich ein Blutbild angefertigt worden. Darin zeigten sich folgende Werte:

- Hb: 10,6g/dl (Referenz: 11,5-16,4g/dl bei Frauen)

- Hämatokrit: 30\% (Referenz: 35-45\%)

- MCV: $69 \mathrm{fl}$ (Referenz: 76-88 fl)

- Eisen: $15 \mathrm{mg} / \mathrm{dl}$ (Referenz: 23-163 mg/dl)

- Ferritin: $30 \mu \mathrm{g} / \mathrm{dl}$ (Referenz: 23-110 $\mu \mathrm{g} / \mathrm{dl}$ )

\section{TABELLE 1}

\section{Differenzialdiagnostische Überlegungen bei eher unspezifischen Allgemeinsymptomen (Auswahl)}

\begin{tabular}{|l|l|}
\hline Symptom & Auswahl möglicher Erkrankungen \\
\hline Müdigkeit, Erschöpfung, Adynamie & $\begin{array}{l}\text { Anämie, Schlafstörungen, Intoxikationen, Tumorerkrankungen, Infektionserkrankungen, Nährstoff- } \\
\text { mangel, psychische Erkrankungen, Hormonstörungen, Hypotonie }\end{array}$ \\
\hline Dyspnoe & $\begin{array}{l}\text { pulmonal: Asthma, COPD, Tumorerkrankungen; kardial: Herzinsuffizienz, Herzklappenerkrankungen; } \\
\text { skelettal: Thoraxblockaden; neuromuskulär: ALS, MS, Poliomyelitis; psychogen: Depression, Stress, } \\
\text { Angst; sonstiges: Anämie, Azidose, Adipositas, Intoxikation }\end{array}$ \\
\hline Tachykardie & $\begin{array}{l}\text { Anstrengung, Anämie, Sinusknotenerkrankungen, Medikamente, Intoxikationen, vermehrte Diurese, } \\
\text { übermäßiges Schwitzen, Mineralstoffverlust }\end{array}$ \\
\hline Vertigo & $\begin{array}{l}\text { Hypotonie, Bewegungsmangel, Hypertonie, Innenohrerkrankungen, Anämie, kardiologische Erkran- } \\
\text { kungen, Alter, Sehstörungen, Durchblutungsstörungen }\end{array}$ \\
\hline Kopfschmerzen & $\begin{array}{l}\text { Migräne, Verspannungen, Trigeminusreize, Hormonstörungen, Nährstoffmangel, Intoxikation, Anä- } \\
\text { mie, Stoffwechselstörungen, Traumata, Durchblutungsstörungen, Infektion, Tumorerkrankungen, } \\
\text { Sinusitis, psychische Ursachen, Neuralgien }\end{array}$ \\
\hline Konzentrationsstörungen & $\begin{array}{l}\text { Bewegungsmangel, Anämie, Schmerzsyndrome, Stoffwechselerkrankungen, Vitalstoffmangel, Schlaf- } \\
\text { störungen }\end{array}$ \\
\hline
\end{tabular}


- Transferrinsättigung (TFS): 186 mg/dl (Referenz: 200-360 $\mathrm{mg} / \mathrm{dl}$ )

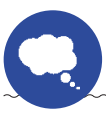

$\rightarrow$ Mit einem $\mathrm{Hb}$-Wert von 10,6 g/dl, einem Hämatokrit von $30 \%$ und einem Eisenwert von $15 \mu \mathrm{g} / \mathrm{dl}$ ist die Diagnose Anämie bestätigt. Noch nicht bewiesen ist damit allerdings, dass es sich dabei auch um die (alleinige) Ursache der Beschwerden handelt. Wodurch die Anämie entstanden ist, liegt ebenfalls noch im Dunkeln.

Wie nicht anders zu erwarten, verordnete der behandelnde Arzt ein Eisenpräparat. Unter dessen Einnahme wurden die Symptome auch bald deutlich besser. Doch schnell traten massive Nebenwirkungen auf, darunter eine für Sophie P. nicht tolerierbare Obstipation. Sobald sie das Mittel absetzte, verschwand diese, doch traten die ursprünglichen Probleme erneut auf. Etwas stimmte nicht.

Weitere Blutbilder waren angefertigt worden: Die Laktatdehydrogenase (LDH) war deutlich erhöht (318U/1; Referenz: 90250 U/1). Bilirubin und Kreatinin lagen im oberen Referenzbereich (Bilirubin: 1,1 mg/dl, Referenz: < 1,1; Kreatinin: 1,1 mg/dl, Referenz 0,8-1,2 mg/dl). Ein Hinweis auf eine hämolytische Anämie ergab sich aus der leichten Erhöhung der Retikulozyten (18 pro 1000 Erythrozyten; Referenz: bis 15/1000).

Glücklicherweise waren die autoimmunologisch relevanten Werte unauffällig. Auch der Hormonstatus fiel sehr gut aus. Außerdem wurde Vitamin $\mathrm{B}_{12}$ in seiner aktiven Form, dem HoloTranscobalamin, bestimmt. Mit einem Wert von 623 ng/l (Referenz: > $400 \mathrm{ng} / \mathrm{l}$ ) konnte ein Mangel ausgeschlossen werden.

\section{Paradoxon: Ursache der Anämie nicht aufzufinden}

Weitere anamnestische Fragen waren erforderlich, um die Ursache entsprechend einzugrenzen. Nach einer etwaigen Blutungsproblematik, unter anderem enterologisch, gynäkologisch und nephrologisch, hatte sich schon der Hausarzt erkundigt. Zudem erklärte Sophie P., dass sie keine Regelblutung mehr habe. Diese sei früher immer pünktlich („wie ein Uhrwerk“) gekommen. Eine Urinuntersuchung hätte allerdings Spuren von Blut im Urin (Mikrohämaturie) ergeben. Die junge Frau verneinte auch etwaige Teerstühle, Hämorrhoidalblutungen oder Magenulcera im Vorfeld.

$\rightarrow$ Die Erwähnung der Mikrohämaturie lässt mich aufhorchen. Allerdings dürfte diese keine so massiven Anämiesymptome auslösen. Da auch sonst keine Hinweise auf ein Blutungsgeschehen vorliegen, stelle ich diese Möglichkeit trotz Erhöhung der Retikulozyten zurück.
Medikamente hatte sie im Vorfeld der massiven Probleme nicht eingenommen. Auf mein Nachfragen, warum sie keine Regelblutung mehr habe, erklärte sie mir, dass ihre Gynäkologin die Amenorrhö auf den Sport zurückgeführt und als „völlig normal“ eingeordnet hätte. Ohne Menses könne sie nun auch ungehindert sportlich aktiv sein.

\section{Differenzialdiagnose: Was könnte außerdem beteiligt sein?}

Nach wie vor galt es, die Ursache der Anämie aufzufinden. Die Abweichungen der $\mathrm{MCH}$ - und MCV-Werte fielen zu gering aus, um daraus weitere Schlüsse zu ziehen. Thrombo- und Leukozyten lagen im Referenzbereich.

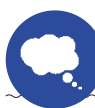

$\rightarrow$ Für die Differenzialdiagnose bei Anämie ergeben besonders die Erythrozytenindizes wichtige Hinweise. So denkt man bei einer deutlichen Verminderung von MCV und MCH an eine Eisenmangelanämie, zum Beispiel durch zu geringe Eisenaufnahme bei vegetarischer oder veganer Ernährung. Eine Makrozytose weist hingegen auf einen Vitamin- $B_{12}$ - oder Folsäuremangel hin. Doch sind bei Sophie P. die Abweichungen dieser Blutwerte sehr gering (MCH: 25,9 pg, Referenz: 26,7-32,8 pg) und damit nur wenig wegweisend. Eine Thrombopenie, Leukozytose oder Leukopenie würde Hinweis auf eine Knochenmarkthematik liefern.

Im Grunde war nun ein optisch-mikroskopisches Differenzialblutbild erforderlich. Spezielle Erythrozytenformen hätten weitere Ursachenmöglichkeiten aufgezeigt. So können sich hier unter anderem Infektionen, Gendefekte (zum Beispiel Targetzellen bei Thalassämie) oder Tumorgeschehen (zum Beispiel Drepanozyten) zeigen.

\section{Spurensuche: Gastritis, Autoantikörper, Fehlernährung oder Gendefekt?}

Welche Ursachen könnten sonst beteiligt sein? Nun lag es an mir, eine anamnestische Liste mit Sophie P. abzuarbeiten. Eine Gastritis könnte die Aufnahme von Eisen, Vitamin $B_{12}$ oder Folsäure behindern. Sie konnte jedoch keines der klassischen Symptome bestätigen. Sowohl eine Autoimmungastritis als auch das Auftreten von Kälte- oder Wärme-Autoantikörpern können ebenfalls zu einer Anämie führen. Doch bis auf kalte Finger im Winterhalbjahr deutete auch hier nichts darauf hin. Auf Fragen zur Ernährung gab sie an, sich gesund und ausgewogen zu ernähren und keine Vegetarierin zu sein. Dies lenkte den Fokus auf mögliche genetische Ursachen, doch auch diese bestätigten sich nicht: Ihre Mutter hätte wohl als junge Frau und während der Schwangerschaften einen Eisenmangel entwickelt, sonst sei aber familiär nichts bekannt.

Auch auf primäre Hormonschwankungen ergab sich neben der mutmaßlich sportbedingten Amenorrhö (dies sollte sich im Fol- 
genden bestätigen) kein näherer Hinweis. Im Blutbild des Arztes war zudem die Schilddrüse mit ihren Werten TSH, fT3, fT4, TRAK und MAK ausführlich untersucht worden. Alle Parameter waren vorbildlich. Auch häufige Diarrhöen, die eine Mangelproblematik provozieren könnten, verneinte Sophie P.

Die körperliche Untersuchung ergab abgesehen von den eingangs festgestellten Anämiezeichen ebenfalls nichts Neues. Allerdings fiel ein Tremor auf. Auf Nachfrage erfuhr ich, dass sie diesen schon seit Jahren habe [8].

$\rightarrow$ Langsam fängt ein Verdacht in mir zu keimen an: Könnte es sein, dass Sophie P. an einer Sportleranämie leidet?

\section{Exzessiver Ausdauersport: War die Ursache gefunden?}

Doch eine Frage stand noch aus: Was für eine Sportart sie denn überhaupt ausübe? Mit zunehmender Begeisterung erzählte mir Sophie P., dass sie bisher vor allem Klettern als Leistungssport ausgeübt hätte. Ihr Freund hätte sie jedoch mit dem Marathonfieber angesteckt. Seit etwa zwölf Monaten würde sie nun umfangreich „auf Ausdauer trainieren“. Sie erklärte mir, dass sie nun fünfmal in der Woche zwischen zwei und drei Stunden - manchmal auch länger - laufe und dabei versuche, ihre Zeit immer weiter zu verbessern.

So wie es aussah, kamen hier mehrere Probleme zusammen. Mit hoher Wahrscheinlichkeit litt Sophie P. unter einer sogenannten Sportleranämie. Obwohl der Begriff etwas irreführend ist (weil er nur einen vorübergehenden Zustand über wenige Tage nach extremer körperlicher Belastung beschreibt), war er in diesem Fall wegweisend: Insbesondere Ausdauer- und junge Sportler entwickeln ein erhöhtes Risiko für eine Eisenmangelanämie.
Diese beruht insbesondere auf den Eisenverlusten über Gastrointestinaltrakt, Schweiß und Urin, vor allem bei Extremsportarten wie Marathon und Triathlon. Dabei führen Flüssigkeitsverluste während körperlicher Belastung in Abhängigkeit von Faktoren wie Dauer, Intensität und Konstitution zu einer Hämokonzentration. Die nachfolgende Flüssigkeitsretention bewirkt jedoch eine ein bis fünf Tage anhaltende Zunahme des Plasmavolumens um bis zu $25 \%$. Entsprechend sinken auch relativ die Blutparameter. Ein regelmäßiges Ausdauertraining führt jedoch langfristig zu einer Steigerung der Erythropoese, sodass im Grunde nur eine Pseudoanämie vorliegt: Die fehlenden Erythrozyten werden innerhalb kurzer Zeit wieder gebildet. Gönnt man dem Körper jedoch keinerlei Ruhe, kann es zu einer dauerhaften Eisenmangelanämie kommen. Bei Langstreckenläufern konnten zudem endoskopisch belastungsinduzierte Mikroblutungen zum Beispiel in Form einer Mikrohämaturie nachgewiesen werden.

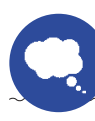

$\rightarrow$ Der Ausdauersport scheint in diesem Fall zu einer Sportleranämie geführt zu haben. Als alleinige Antwort auf alle vorliegenden Symptome und Befunde ist dies jedoch nicht wirklich zufriedenstellend, weil der Organismus eine Sportleranämie normalerweise relativ schnell durch Anpassung an die erhöhte Leistungsanforderung beseitigt. Hier kommt meine zweite Verdachtsdiagnose ins Spiel.

\section{Cofaktor Morbus Wilson erklärt das} Gesamtbild

Einige Zeichen wie Tremor, junges Alter, leicht erhöhte Retikulozyten und Konzentrationsschwierigkeiten lenkten meinen Verdacht nun auf eine weitere Erkrankung: die Kupferspeicherkrankheit Morbus Wilson. 
$\rightarrow$ Typisch für Morbus Wilson ist eine Manifestation der Beschwerden vor dem 20. Lebensjahr. Die typische Kupferaggregation in der Kornea im Sinne eines Kayser-Fleischer-Ringes ist bei Sophie P. zwar nicht erkennbar, muss aber in frühen Stadien noch nicht ausgebildet sein.

Unter anderem kommt es bei der Erkrankung durch den toxischen Einfluss erhöhter Kupferkonzentrationen im Blut häufig zu einer hämolytischen Anämie. Zwei Laborwerte bestätigten dies umgehend: Bei Sophie P. lag ein leicht erniedrigter Serumkupferwert vor (gerade in frühen Phasen der Erkrankung speichert das Bindegewebe das Kupfer, insofern findet sich davon weniger in der Blutbahn). Auch das Coeruloplasmin zeigte sich erniedrigt, was eine relativ sichere Diagnose ergibt. Die Kupferspeicherkrankheit war also ein Cofaktor der vorhandenen Anämie. Für Sophie P. kamen also Eisenverluste durch extreme Ausdauersportbelastung und eine hämolytische Anämie durch Morbus Wilson zusammen.

Merke: Da es sich bei Morbus Wilson um eine genetische Erkrankung handelt, ist eine Heilung oder kausale Therapie nicht möglich.

\section{Therapie: alpha-Liponsäure, Zink, Eisen und Ernährungsumstellung}

Wie konnte nun der therapeutische Ansatz aussehen? Auch hier mussten wir multikausal arbeiten. Sophie P. entschied sich dafür, zunächst auf eine allgemeinmedizinische Therapie des Morbus Wilson mit Chelatbildnern zu verzichten. Dies würde jedoch nur so lange möglich sein, wie sich Leber- und Blutwerte sowie andere Parameter nicht verschlechterten. Eine engmaschige Laborkontrolle war somit essenziell.

Zur Therapie des M. Wilson hat sich zum einen alpha-Liponsäure bewährt, hier: Thiogamma ${ }^{\circledR} 600$ (Fa. Wörwag), $1 \times$ tgl. 1 Tbl.

$\rightarrow$ Die körpereigene alpha-Liponsäure kann Metalle wie Kupfer binden, sie so wasserlöslich machen und eine Ausscheidung über die Niere ermöglichen. Dabei greift sie keine an Proteine gebundenen Substanzen an.

Zum anderen verbessert Zink die Stoffwechsellage, denn es reduziert die Kupferresorption im Darm. Daher verordnete ich Zink$\operatorname{orot}^{\circledR} 25$ (Fa. Wörwag), $1 \times$ tgl. 2 Tbl. Auch die Ernährungsoptimierung ist von besonderer Bedeutung. Sophie P. sollte fortan kupferreiche Lebensmittel wie Schokolade, Sonnenblumenkerne, Hummer, Krabben, Kakao oder Nüsse meiden. Außerdem vereinbarte sie mit dem Gynäkologen einen Termin zur Entfernung ihrer Kupferspirale.
Merke: Da es sich bei alpha-Liponsäure um eine sehr reagible Substanz handelt, muss die Einnahme mindestens 60-90 min getrennt von anderen Mitteln erfolgen. Vor allem Mineralstoffpräparate können bei geringerem Abstand leicht ihre Wirkung verlieren. Auch Eisen und Zink sollten in diesem Abstand eingenommen werden, um nicht um die Resorption zu konkurrieren.

Um die Anämie zu beseitigen, waren ebenfalls mehrere Maßnahmen notwendig. So würde die Patientin ihr Trainingsvolumen reduzieren und ihre Marathonambitionen aufgeben. Sie sollte tgl. 1 Kps. Eisen einnehmen (ferro sanol ${ }^{\circledR}$ comp., Fa. UCB-Pharma), außerdem Vitamin $\mathrm{B}_{12}$ und Folsäure (Vitamin $\mathrm{B}_{12}$ Ankermann Tbl. [Fa. Wörwag], $2 \times$ tgl. 1 Tbl.; Folsäure [Fa. Hevert], $2 \times$ tgl. 1 Tbl.). Diese Kombination optimiert die Aufnahme des Eisens und vermindert obstipative Nebenwirkungen. Um eine Konkurrenz bei der Resorption zu verhindern, sollten Eisen und Zink etwas zeitversetzt eingenommen werden. Außerdem verordnete ich Infifer ${ }^{\circledR}$ (Fa. Infirmarius), $3 \times$ tgl. 15 Tr. Der homöopathische Komplex dient zur Optimierung des Eisenstoffwechsels, ist selbst allerdings kein Substitut.

Auch im Hinblick auf die Anämie war eine optimierte Ernährung wichtig. Eisenhaltige Lebensmittel müssen jedoch grundsätzlich auf ihren Kupfergehalt überprüft werden. Hier stand eine unvermeidbare Recherchearbeit für die Patientin an.

\section{Ergebnis: Deutliche Besserung nach acht Wochen}

Schon nach acht Wochen Therapie ging es Sophie P. deutlich besser: Sie freute sich über einen spürbaren Leistungszuwachs, eine gesündere Hautfarbe und ein deutlich besseres Konzentrationsvermögen. Ihre Vergesslichkeit war verschwunden. Sie war nun zuversichtlich, ihr altes Niveau bald wieder zu erreichen.

Dieser Artikel ist online zu finden:

http://dx.doi.org/10.1055/a-1064-6909

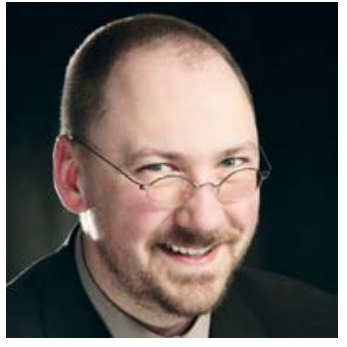

HP CLAUS JAHN

Claus Jahn ist seit über 24 Jahren als Heilpraktiker niedergelassen und arbeitet als freier Mitarbeiter am Lehrund Forschungszentrum „Felke-Institut". Er ist Autor zahlreicher Fachartikel und einiger Bücher. Seit 20 Jahren ist er national und international als Referent zu unterschiedlichen naturheilkundlichen Themen tätig.

E-Mail: c.jahn@naturheilpraxis-jahn.de 